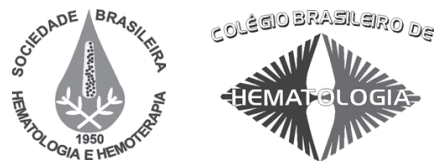

\title{
Osteonecrose maxilar em pacientes portadores de doenças neoplásicas sob uso de bisfosfonatos
}

\author{
Jaw osteonecrosis in patients with neoplastic diseases taking bisphosphonates
}

\author{
Paulo S. S. Santos ${ }^{1}$ \\ Liane M. Gambirazi ${ }^{2}$ \\ Valtuir B. Felix ${ }^{1}$ \\ Marina Helena C. G. Magalhães ${ }^{3}$
}

\begin{abstract}
A osteonecrose induzida por bisfosfonatos é uma complicação que pode ocorrer em pacientes acometidos por doença osteolítica tais como mieloma múltiplo, portadores de metástases tumorais em tecido ósseo, osteoporose e que fizeram uso de droga do grupo dos bisfosfonatos. A despeito dos beneficios do uso destes fármacos, a osteonecrose maxilar é uma importante complicação. Seu mecanismo de ação reduz a reabsorção óssea, o estímulo à atividade osteoblástica, a inibição do recrutamento e promoção da apoptose de osteoclastos. Até o presente momento, não há na literatura um protocolo de tratamento para a osteonecrose por bisfosfonatos. No presente trabalho, os autores fazem uma revisão da literatura e descrevem dois casos clínicos em pacientes do sexo feminino, com diferentes doenças, mieloma múltiplo e metástases ósseas por carcinoma de mama, acometidas por osteonecrose em mandibula induzida por bisfosfonatos. Rev. Bras. Hematol. Hemoter. 2008;30(6):501-504.
\end{abstract}

Palavras-chave: Osteonecrose; bisfosfonatos; doenças maxilares.

\section{Introdução}

Atualmente há a preocupação quanto ao uso dos bisfosfonatos (BFs) na necessidade de manipulação de tecidos gengivais e ósseos. Badros (2006) observou em pacientes com mieloma múltiplo (MM) tratados com BFs nove vezes maior propensão em desenvolver osteonecrose nos que fizeram extrações dentárias e aumento de $9 \%$ no risco de desenvolver osteonecrose a cada década de envelhecimento dos pacientes. Na correlação de osteonecrose e BFs, concluiu que cada ano vivido com o MM aumentou em 57\% o risco de desenvolver osteonecrose. ${ }^{1}$ De relevância semelhante, o osso é o local mais comum de doença metastática associada com câncer de mama, afetando mais da metade das mulheres durante o curso da doença. BFs que inibem osteoclastos mediados por reabsorção óssea têm sua aplica- ção na redução da dor óssea e melhora da qualidade de vida. ${ }^{2}$

São fatores de risco para osteonecrose induzida por bisfosfonatos (ONB): extração dentária concomitante ao uso de bisfosfonatos, terapia com pamidronato/ácido zoledrônico (BFs), idade do paciente e tempo de diagnóstico da doença. ${ }^{3}$

Embora a etiologia deste processo osteonecrótico permaneça obscura, várias drogas para tratamento do câncer podem induzir osteonecrose e algumas delas são comumente usadas em associação com os BFs. Destacam-se os corticóides, talidomida, vincristina, ciclofosfamida e metotrexato as quais têm efeitos antiangiogênicos, como inibição do VEGF e do fator de crescimento fibroblástico. ${ }^{4,5,6}$

Neste artigo relatamos o manejo clínico e cirúrgico de dois casos de pacientes oncológicos que fizeram uso de BFs e desenvolveram osteonecrose nos maxilares, sendo diagnosticados como ONB.

\footnotetext{
${ }^{I}$ Cirurgião-dentista do Centro de Atendimento a Pacientes Especiais da Faculdade de Odontologia da Universidade de São Paulo - Cape/FouspSão Paulo-SP

${ }^{2}$ Cirurgiã-dentista da Oncoclin - São Paulo-SP.

${ }^{3}$ Professora Titular da Disciplina de Patologia Bucal da Faculdade de Odontologia da Universidade de São Paulo (Fousp) - São Paulo-SP.
}

Faculdade de Odontologia da Universidade de São Paulo (Fousp) - São Paulo-SP.

Correspondência: Paulo Sérgio S. Santos

Av. Professor Lineu Prestes, 2227, Cidade Universitária

05508-000 - São Paulo-SP

Email: paulosergiosilvasantos@gmail.com 


\section{Casos Clínicos}

Caso 1: Paciente de 69 anos, sexo feminino, raça amarela, com MM sob adequação bucal prévia ao transplante de medula óssea. Realizou quimioterapia com vincristina, adriblastina e dexametasona (VAD) e bisfosfonato $4 \mathrm{mg} / \mathrm{mês}$ (Zometa ${ }^{\circledR}$ ) durante nove meses. Apresentava periodontite em vários dentes e exposição óssea na região de trígono retromolar à direita, com $0,2 \mathrm{~mm}$ de diâmetro. Havia múltiplas imagens radiolúcidas nas radiografias panorâmica e de crânio, compatíveis com MM. Aúltima sessão de quimioterapia e bisfosfonato havia sido realizada quarenta dias antes do procedimento cirúrgico, quando a paciente apresentava hemograma com parâmetros de normalidade. A pequena área de osso com aparência necrótica foi removida, sob antibioticoterapia, e a análise histológica demonstrou osso necrótico. Não houve reparação tecidual após a biópsia. Realizada nova intervenção cirúrgica 45 dias após as exodontias, com finalidade de decorticar o osso exposto e arredondar as bordas cortantes, facilitando a coaptação das bordas gengivais. Houve boa reparação 15 dias após esta intervenção. A paciente foi acompanhada por três meses, a mucosa permaneceu íntegra neste período (Figuras 1,2 e 3) e foi reabilitada com próteses totais.

Caso 2: Paciente do sexo feminino, leucoderma, 57 anos, apresentou carcinoma de mama (1994), quando realizou mastectomia radical, quimioterapia CMF (ciclosfosfamida, metrotexate e fluorouracil) e radioterapia. Em 2000 apresentou metástases ósseas, realizou quimioterapia com FAC (fluorouracil, adriblastina e ciclosfosfamida), seis ciclos, radioterapia em coluna lombo-sacra e iniciou Arédiaß. Em 2003 evoluiu com metástase hepática, realizou quimioterapia com taxotere, seis ciclos, substituiu Arédia ${ }^{\circledR}$ pelo Zometa ${ }^{\circledR}$ e iniciou hormonioterapia com Zoladex ${ }^{\circledR}$ e Lentaron (formestano), substituídos pelo Femara ${ }^{\circledR}$ (bloqueando a produção de estrógenos) e atualmente utilizando Aromasin ${ }^{\circledR}$. Em agosto de 2006, a paciente queixou de dor em mandíbula esquer$\mathrm{da}$; foi observada discreta fístula em rebordo gengival inferior, na região dos pré-molares esquerdos. Em dezembro de 2006 houve piora da dor, mantendo fístula com exsudato. Devido à infecção iniciou antibioticoterapia com clindamicina durante 14 dias.

O controle da infecção resultou em melhora da dor. Em março de 2007 houve aumento volumétrico no local, com apagamento do sulco vestibular. A mucosa apresentava coloração normal, fístula com exsudato, resiliente à palpação e com sintomatologia dolorosa. As radiografias panorâmica e periapicais revelavam imagem radiolúcida difusa, e a tomografia revelou expansão óssea sem rompimento da cortical. A cintilografia óssea mostrou hipercaptação em mandíbula à esquerda. Realizada biópsia incisional de tecido mole e duro, com hipóteses diagnósticas de ONB ou metástase tumoral em mandíbula (Figuras 1e 2). O exame histopatológico reve-
CASO 1

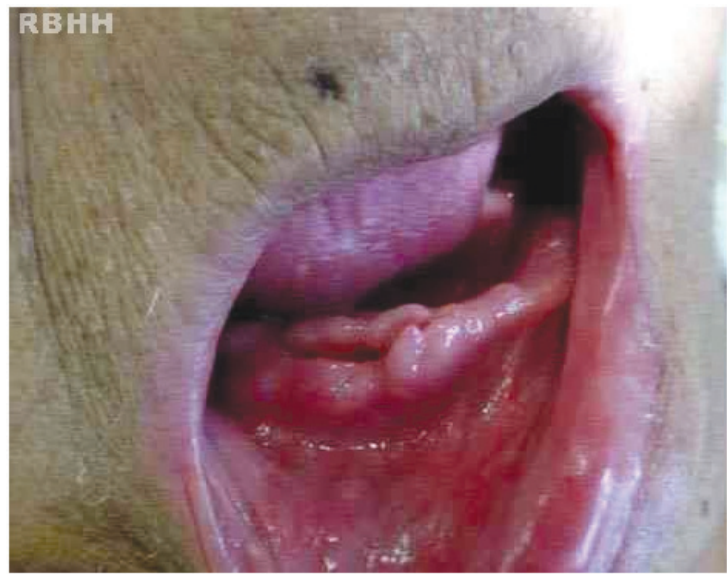

Figura 1. Região onde foram realizadas as exodontias

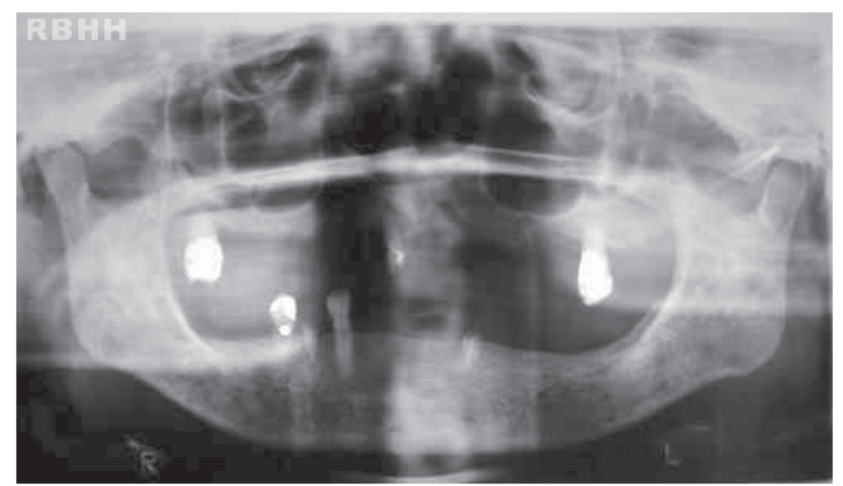

Figura 2. Radiografia panorâmica evidenciando padrão ósseo misto com áreas radiolúcidas características do mieloma múltiplo

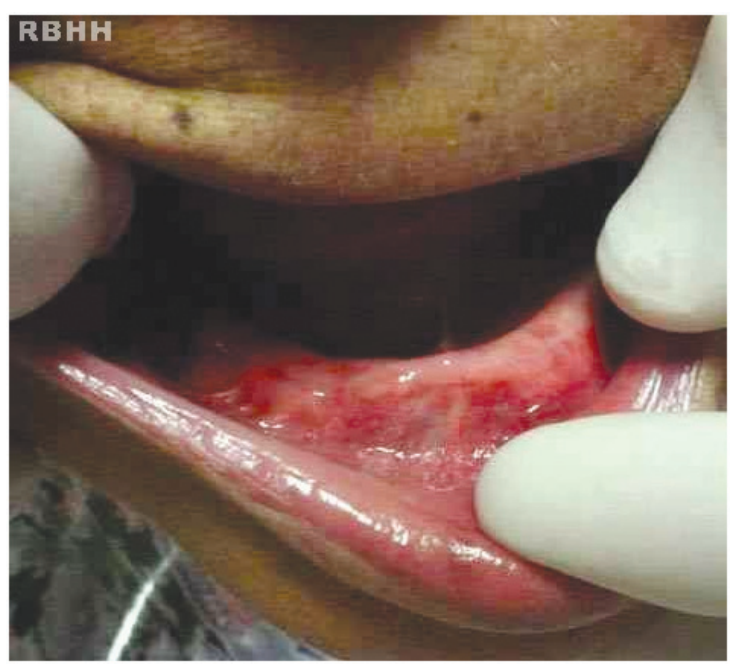

Figura 3. Região de reparo tecidual completo após manipulação cirúrgica (3 meses) 
CASO 2

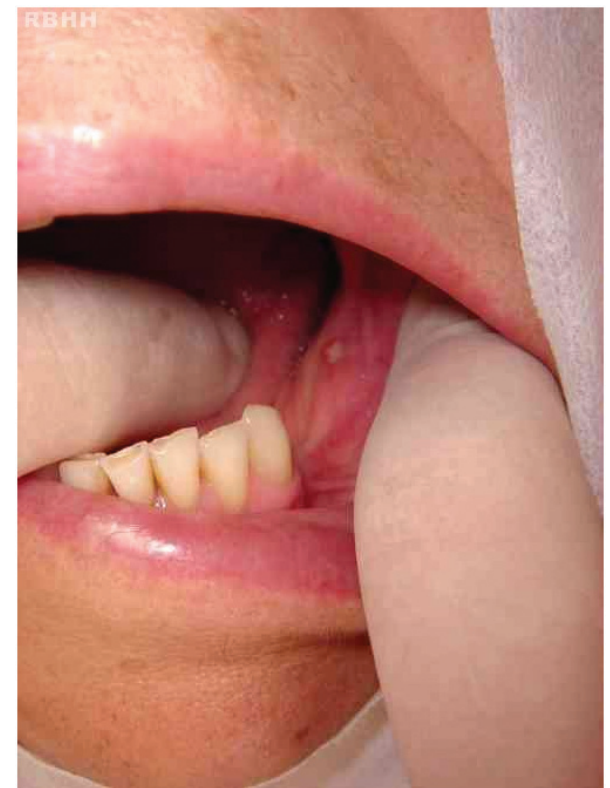

Figura 1. Área de exposição óssea em rebordo mandibular

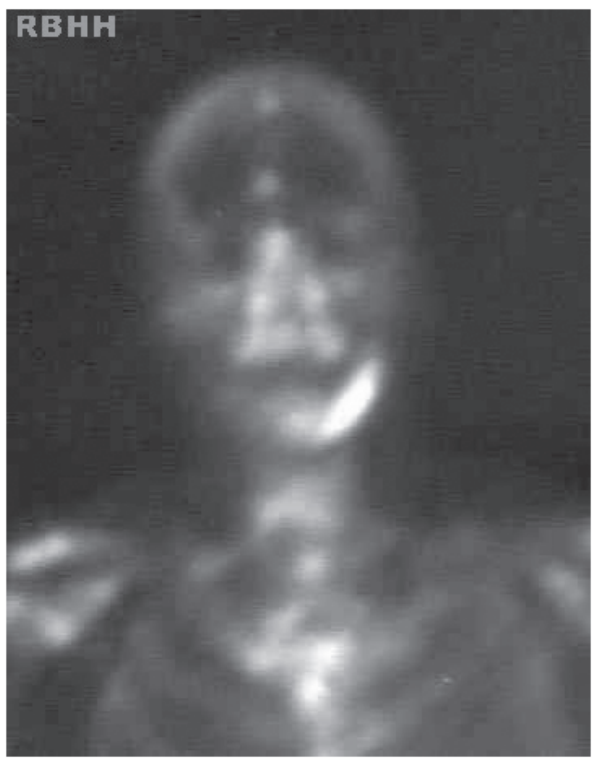

Figura 2. Cintilografia óssea evidenciando área hipercaptante em corpo de mandíbula esquerda

lou processo inflamatório crônico e agudo e osso necrótico. O diagnóstico final foi de osteonecrose. Não houve cicatrização no local, persistindo a exposição óssea com aproximadamente $2 \mathrm{~cm}$ de extensão. Os quadros de dor são esporádicos. O bisfosfonato foi suspenso em abril de 2007, após o diagnóstico de osteonecrose. Paciente encontra-se em acompanhamento e em programação de nova intervenção cirúrgica com debridamento no local e uma abordagem mais agressiva.

\section{Discussão}

Dentre os fatores de risco para ONB destacam-se: as exodontias concomitantes ao uso dos bisfosfonatos (BFs), terapia com pamidronato/ácido zoledrônico, idade do paciente e tempo de diagnóstico da doença. ${ }^{3}$ Pacientes que fizeram extrações dentárias são mais propensos a desenvolver osteonecrose e, com o passar do tempo pós-extração, aumenta ainda mais o risco de desenvolver ONB, sendo que mais de $57 \%$ dos pacientes com mieloma múltiplo desenvolvem osteonecrose. ${ }^{1}$ No caso 1 , a lesão de ONB surgiu espontaneamente sem história de trauma, e em região edêntula, em paciente de 69 anos sob uso do ácido zoledrônico (Zometa ${ }^{\circledR}$ ) administrado mensalmente há nove meses. Na série de Marx, ${ }^{7}$ a maioria dos pacientes que desenvolveram ONB usou pamidronato $(67 \%)$, enquanto o restante $(33 \%)$, o ácido zoledrônico. Ruggiero et al também relataram em sua amostra que $74,6 \%$ usaram pamidronato, e $36,4 \%$, o zoledronato.

A etiopatogenia da osteonecrose continua em investigação, mas a relação entre o uso de BFs e o desenvolvimento de necrose óssea em tecidos bucais após manipulação ou trauma tem ficado cada vez mais clara. A doença de base dos pacientes parece não ter tanta relevância na incidência, o que exemplificamos mostrando que pacientes com diferentes diagnósticos, mas utilizando BFs, desenvolveram a ONB.

O tratamento da ONB continua muito variável na literatura incluindo múltiplas abordagens: ${ }^{6,9,10}$

$\checkmark$ Conservadoras: debridamentos dos sequestros ósseos seguidos por irrigação com soro fisiológico e bochechos diários com clorexidina $0,12 \%$. Pode ser associado a analgésicos e antimicrobianos. ${ }^{11}$

$\checkmark$ Radicais: ressecções com reconstruções primárias ou secundárias.

A oxigenoterapia hiperbárica, para muitos autores, ainda tem ação controversa, uma vez que não é uniformemente efetiva em diminuir a progressão do processo de osteonecrose avascular. $^{5}$

Em 2006, Ruggiero et al. ${ }^{2}$ recomendaram um protocolo de tratamento de acordo com o estadiamento clínico da ONB que, no estágio I (exposição com osso necrótico e assintomático), deverão ser realizados bochechos diários com solução antimicrobiana (clorexidina a $0,12 \%$ ) e acompanhamento regular do paciente. No estágio 2 (exposição associada à osso necrótico com dor e infecção) terapia antimicrobiana baseada em cultura e antibiograma, analgesia e bochechos diários ou irrigações com clorexidina, e no estágio 3 (exposição com osso necrótico, dor, infecção e fratura patológica com fístula extra-oral ou osteólises se estendendo a basal mandibular) debridamento cirúrgico dos sequestros ósseos, terapia antimicrobiana (EV ou VO), analgesia e bochechos diários ou irrigações com clorexidina a $0,12 \%$.

No caso 2 (metástase óssea), a paciente referia dor discreta, trismo e havia pequena exposição de osso necrótico (estágio 2$),{ }^{2}$ e o tratamento consistiu no debridamento do 
osso desvitalizado, antibioticoterapia, analgésicos e bochechos diários com clorexidina a $0,12 \%$. Estes cuidados com redução de biofilme microbiano em relação à ONB são considerados de fundamental importância por Sedghizadeh. ${ }^{5}$ No entanto, não obtivemos sucesso nas condutas realizadas.

As orientações aos pacientes com maior risco de desenvolver ONB são fundamentais. Estes pacientes devem ser avaliados criteriosamente por médicos e dentistas, especialmente aqueles que estão em uso da droga intravenosa.

A indicação da suspensão dos BFs em caso de ONB até o momento tem sido uma medida empírica, não se conhecendo ainda que importância existe em relação à reparação da exposição óssea.

\section{Conclusões}

Com o número crescente de casos de ONB, a natureza não remissiva da lesão e a morbidade associada, os pacientes que recebem BFs devem ser orientados quanto à possibilidade de desenvolver a doença, antes de iniciar o tratamento, principalmente pamidronato e ácido zoledrônico, devendo também ser orientados a realizar tratamento odontológico prévio.

O tratamento da ONB tem como consenso uma abordagem multiprofissional. A suspensão ou substituição dos BFs deverá ser bem avaliada quanto ao custo-benefício no bemestar do paciente e requer a realização de estudos prospectivos que demonstrem suas vantagens e desvantagens.

\section{Abstract}

The use of bisphosphonates among patients affected by osteolytic diseases, such as multiple myeloma, metastatic bone lesions and osteoporosis has been associated with the risk of osteonecrosis of the jaws. Bisphosphonates are found in areas of the bone that are undergoing inflammation or resorption. They are phagocytosed and internalized by osteoclasts. Once in the bone, these bisphosphonates cause apoptosis or cell death of the osteoclasts and as a result they may inhibit osteoclast-mediated bone resorption. Bisphosphonates seem to affect osteoclasts when it comes to both numbers and function. Although bisphosphonates are potent and valuable inhibitors of osteoclastic bone lesions, several unanswered questions exist regarding the risk of developing osteonecrosis and the management of this complication. This study reports two clinical cases of osteonecrosis of the jaws associated with the use of bisphosphonates. According to the findings, the two patients (women) with different neoplasms: multiple myeloma and breast cancer with metastatic bone lesions presented some signs and symptoms of osteonecrosis. Rev. Bras. Hematol. Hemoter. 2008;30(6):501-504.

\section{Referências Bibliográficas}

1. Badros A, Weikel D, Salama A, Goloubeva O, Schneider A, Rapoport A et al. Osteonecrosis of the jaw in multiple myeloma patients: clinical features and risk factors. J Clin Oncol. 2006; 24(6):945-52.

2. Ruggiero SL, Fantasia J, Carlson E. Bisphosphonate-related osteonecrosis of the jaw: background and guidelines for diagnosis, staging and management. Oral Surg Oral Med Oral Pathol Oral Radiol Endod. 2006;102(4):433-41.

3. Schwartz HC, Kagan AR. Osteoradionecrosis of the mandible: scientific basis for clinical staging. Am J Clin Oncol. 2002;25(2): 168-71.

4. Bamias A, Kastritis E, Bamia C, Moulopoulos LA, Melakopoulos $\mathrm{I}$, Bozas $\mathrm{G}$ et al. Osteonecrosis of the jaw in cancer after treatment with bisphosphonates: incidence and risk factors. J Clin Oncol. 2005;23(34):8580-7.

5. Sedghizadeh PP, Kumar SK, Gorur A, Schaudinn C, Shuler CF, Costerton JW. Identification of microbial biofilms in osteonecrosis of the jaws secondary to bisphosphonate therapy. J Oral Maxillofac Surg. 2008;66(4):767-75.

6. Zervas K, Verrou E, Teleioudis Z, Vahtsevanos K, Banti A, Mihou $\mathrm{D}$ et al. Incidence, risk factors and management of osteonecrosis of the jaw in patients with multiple myeloma: a single-centre experience in 303 patients. Br J Haematol. 2006;134(6):620-3.

7. Marx RE. Pamidronate (Aredia) and zoledronate (Zometa) induced avascular necrosis of the jaws: a growing epidemic. J Oral Maxillofac Surg. 2003;61(9):1115-7.

8. Ruggiero SL, Mehrotra B, Rosenberg TJ, Engroff SL. Osteonecrosis of the jaws associated with the use of bisphosphonates: a review of 63 cases. J Oral Maxillofac Surg. 2004;62(5):527-34.

9. Bagan JV, Murillo J, Jimenez Y, Poveda R, Milian MA, Sanchis JM, et al. Avascular jaw osteonecrosis in association with cancer chemotherapy: series of 10 cases. J Oral Pathol Med. 2005;34 (2):120-3.

10. Pavlakis N, Schmidt R, Stockler M. Bisphosphonates for breast cancer. Cochrane Database Syst Rev. 2005;(3):CD003474.

11. Merigo E, Manfredi M, Meleti M, Corradi D, Vescovi P. Jaw bone necrosis without previous dental extractions associated with the use of bisphosphonates (pamidronate and zoledronate): a fourcase report. J Oral Pathol Med. 2005;34(10):613-7.

Avaliação: Editor e dois revisores externos Conflito de interesse: não declarado

Recebido: 01/06/2008

Aceito após modificações: 06/10/2008

Key words: Osteonecrosis; bisphosphonates; maxillary diseases. 